\title{
Advances in the Development of Tissue Engineering Applied to the Skin Using 3D Bio-Printers for the Treatment of Burn Patients
}

\author{
Giovanni Alcocer*, Priscilla Alcocer \& Alberto Bolgiani \\ Independent Researchers, Guayaquil-Ecuador.Email: giov_alc_science@hotmail.com* \\ Giovanni Alcocer: Master in Physics with specialization in Astrophysics and Medical Physics, Professor of Physics, Advanced Mathematics and Science in \\ general, Author of the recognized and renowned articles: The Fundaments of the Mass: Gravitation, Electromagnetism and Atom.
}

DOI: http://doi.org/10.46382/MJBAS.2021.5104

Copyright: (02021 Giovanni Alcocer et al. This is an open access article distributed under the terms of the Creative Commons Attribution License, which permits unrestricted use, distribution, and reproduction in any medium, provided the original author and source are credited.

In 1975, Dr. Burke and Dr. Yahanas (at MGH and MIT) were the first to develop an idea and prototype of an artificial dermis. In the year of 1976, Dr. Green (at MGH) got a sheet of keratinocytes in vitro. In the year of 1980, there is the clinical use and improvement of the technique. In the year of 1982, a laminar system of cells is achieved. In 2000, the development in vitro of the dermis and epidermis is achieved. In 2010, the developmen of $3 D$ skin printers with the ability to print the dermis and epidermis with the cells of the patient with thickness control and printing area is produced ${ }^{30}$. The concept of $3 D$ printing was developed in 1980. The idea of $3 D$ printing to manufacture objects was established by Charles Hull in the year of $1986^{4}$. His idea was that successive layers of a base material could be added at the top of each to manufacture (print) objects. The first $3 D$ printing was designed by Sachs in 1993 to print plastics and metals. Then, a series of $3 D$ printers have been developed with different applications. Besides, in 2002 (Italy), another dermal regeneration matrix (hyalomatrix) was developed with hyaluronic acid and silastic fibers, mimicking the epidermis. In 2003 (Mexico) an allogeneic keratinocyte culture is developed as a system for the release of growth factors in skin lesions. In the last 10 years, 15 matrices of dermal regeneration have appeared ${ }^{30}$.

It has had a great impact in engineering and medicine. In the medical field, a very important application is the tissue engineering, not only for the manufacture of skin and grafting but also for conducting scientific experimentation in the evaluation and discovery of drugs ${ }^{l}$. Bio-printing of tissues can also help to the study of skin disorders and diseases. ${ }^{1}$. By using $3 D$ bio-printing, the respective aggregation layer by layer of the cells is obtained. It allows the organization of multiple cell types in a desired structure. Then, the respective cell culture is performed in vitro ( 3 to 4 weeks), allowing the respective growth and maturation to achieve the desired tissue. Thus, the tissue implantation is performed.

The conventional methods of tissue engineering (without $3 D$ bio-printers) have little spatial relationship between the individual elements (cells) of the desired tissue ${ }^{4}$. For other hand, the $3 D$ bio-printing technique improves both spatial resolution and reproducibility. Therefore, it is possible to obtain the optimal conditions for cell incubation and maturation.

Besides, organ transplantation is one of the biggest treatments in medicine for many organ disorders. However, the supply of donors is limited and thus, the biofabrication of organs and tissues can help for the respective transplant ${ }^{5}$.

\section{Introduction}

The skin is very important to maintain homeostasis and to provide protection from the external environment ${ }^{1}$. It provides a barrier to the entry of xenobiotics into the body. Also, it regulates the transport of water and metabolites outside the body.

The 3D engineering applied to the skin has advanced along with the development of techniques and models in vitro (biological techniques).

The most basic model to represent the skin is to use two layers: epidermis and dermis.

\section{Cell culture, Methods and Materials}

The keratinocytes and fibroblasts can be used as constituent cells to represent the epidermis and dermis respectively. For other hand, collagen can be used to represent the dermal matrix of the skin (the scaffold structure) $)^{1}$. There are printing techniques that print together with the scaffold (or dermal matrix) the cellular material. Other techniques print the scaffold firstly, and then the cellular material is added. Currently, the hydrogel 
is under experimentation to represent this dermal matrix. The dermal matrix is the base impression material where the keratinocytes and fibroblasts are added. As it was mentioned before, the most basic structure to represent the skin is two layers: epidermis and dermis. The epidermis is represented by the keratinocytes and the stratum corneum is represented by corneocytes. The dermis is represented by synthetic substrates (nylon and polycarbonates) or protein stratifications (such as collagen, glucosamine and fibrin) or dermis from dead skin or fibroblasts. Fibroblasts is the most used to represent the dermis among these substrates.

The incubation technique consists in growing (culturing) keratinocytes-corneocytes in an air-liquid interface in a dermal substitute ${ }^{1}$. It is a process of incubation and exposure of the epidermis to an air-liquid interface to achieve maturation and stratification of the $\operatorname{skin}^{1}$. Also, it helps the formation of the stratum corneum ${ }^{1}$.

The keratinocytes receive nutrients from the lower surface of the culture in a culture period of 3-4 weeks ${ }^{1}$. The culture medium needs to be changed sometimes during this period ${ }^{1}$. The cell viability and cell interactions can be analyzed through a microscope ${ }^{1}$. In this way, it is possible to obtain a tissue that mimics the biological, physiological and morphological characteristics of the human skin.

Also, this model is very good for reproducing the structure of the skin. However, it must be improved in regards to the cellular interaction (coupling) and immune functions of the skin (functionality). For other hand, it is necessary to improve the yield of these crops. In addition, 3D engineering allows us to obtain a stratified matrix where several tissues can be manufactured according to the respective function and different $\operatorname{sizes}^{1}$. One of the techniques for culturing the cells is the following: fibroblasts and keratinocytes (it was added trypsin and kept on ice before printing) can be grown at a temperature between 35 and 40 degrees Celsius, with a solution of $\mathrm{CO}_{2}$, penicillin and bovine serum ${ }^{1}$.

Among the additive factors which are added to improve the tissue maturation are: human keratinocyte supplement, hydrocotrisone, bovine insulin, ascorbic acid, calcium chloride. Besides, the medium is changed during culture every day for approximately two weeks ${ }^{1}$. Hydrogel (it was diluted with a phosphate-saline solution and kept on ice before printing) is used as a collagen and as a scaffold material for printing ${ }^{1}$.

The inkjet pressure (ranges from 1 to 3 psi) and the duration of the printing pulses (500 to $1000 \mu$ s) (separation between cells: 100 to $1000 \mu \mathrm{m}$ ) are important for the cell viability, resolution, droplet volume (20 to $60 \mathrm{~nL}$ ) and cell interaction. These parameters are determined from the viscosity of the biomaterials that are printed ${ }^{1}$.


Fig.1. Printing scheme of the layer by layer method

Firstly, two layers of collagen were printed. Then a layer of fibroblasts and/ or keratinocytes was printed. The last figure corresponds to a top view of the layers ${ }^{1}$. This process can be repeated several times. Thus, it is possible to get the following scheme: 


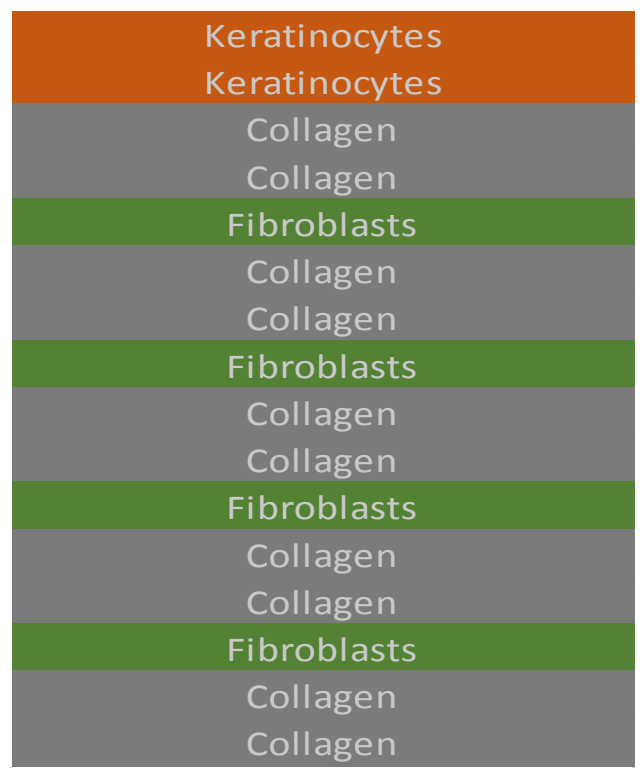

Fig.2. Construction of the 3D tissue of the layer by layer method

Here, 10 layers of collagen, 4 layers of fibroblasts (dermis) and two layers of keratinocytes (epidermis) have been used. In addition, layers of corneocytes can be included to represent the stratum corneum and other additive factors to improve the culture in conjunction with the fibroblasts or keratinocytes ${ }^{1}$.

Sodium bicarbonate vapor can be applied at the interface of each collagen layer and between the first collagen layer and the support glass disk. It is done to increase adhesion between the layers ${ }^{1}$.

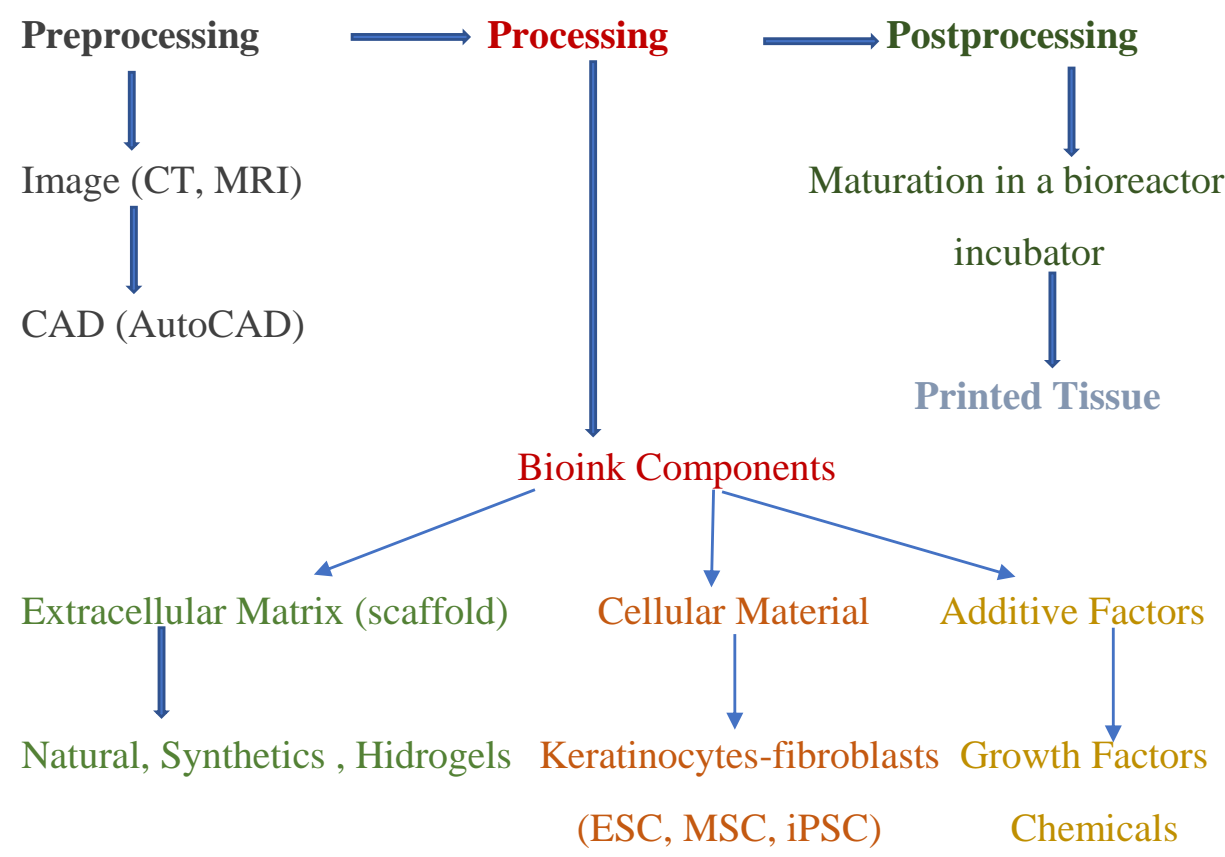

Fig.3. Bioprinting scheme ${ }^{4}$

\section{3D Bioprinters Applied to Tissues}

Preliminary studies and analyzes can be carried out through this engineering, to obtain the optimal printing parameters (pressure, speed and vertical movement ( $\mathrm{z}$ offset of the injector). It would give rise to appropriate 
results for cell viability and densities, concentrations ( 1 to $5 \mathrm{mg} / \mathrm{mL}$ for Collagen, 0.5 to 10 million cells $/ \mathrm{mL}$ for Cell suspension) and percentages cells in the epidermis and dermis. Besides, it would serve as equivalents of the skin in the physiological and anatomical aspect ${ }^{1}$.

It is also an advantage with respect to the use of animal skin to mimic the physiology of the skin (due to ethical aspects) and to study skin disorders. Besides, it is an advantage over conventional tissue grafts without the use of the 3D printer with regard to reproducibility and high crop yield levels. Therefore, the optimal printing parameters for cell viability and proliferation such as resolution and cell density can be obtained by using the 3D printer.

There are the following printing parameters: air pressure (inkjet), pulses of printing duration (for inkjet/drop printing), size of the printing layer, volume of the drop, space between drops/resolution, cell density ${ }^{1}$.

The resolution of the bio-printing techniques ranges from a scale of a few micrometers to a little beyond $100 \mu \mathrm{m}^{3}$. Actually, the types of printing are: lithography, laser, ink-jet deposition, and photopolymerization ${ }^{3}$.

There are the next strategies used in tissue bioprinting: biomimicry, autonomous assembly, and microtexture ${ }^{4}$.

Another application is to obtain superficial grafts (as bandages) for wound healing (there is also a spray that helps heal superficial wounds) and to study skin disorders. It is important that the bioprinting method does not produce toxicity or collateral damage to the cells and their DNA. Also, the bio-printing method must result in a mechanically and functionally stable tissue. There are two techniques: the one that involves incubation and without incubation (performed in the operating room, clinical bioprinting). There must be cell viability in vitro before the tissue integration with a good specific functioning of the tissue with respect to the technique that involves incubation ${ }^{3}$. Currently, hydrogel as a collagen and printing base material is under study to achieve the development of functional tissues.

For other hand, the cells show different biological activity in 2D and 3D environments. Thus, the printing of tissues and organs require a 3D scheme ${ }^{3}$. It is possible to perform the manual (conventional) assembly of 2D cell sheets or layers (layer by layer printing) to a 3D structure.

The conventional tissue manufacturing involves cell culture within a scaffold that has a porous structure to mimic the properties of the extracellular matrix ${ }^{5}$. This technology using scaffold (extra-cellular matrix) has been used to make bones, skins and cartilage. However, it has complications to mimic complex tissue structures and to locate multiple types of cells in desired and ordered positions ${ }^{5}$.

The future of tissue engineering is the manufacture of functional tissues and organs for the respective transplant in a reasonable time scale ${ }^{3}$. In addition, efficiency, automation, reproducibility, scalability (micrometer resolution) is obtained with the 3D printer. 3D bio-printing can be considered as a simultaneous deposition of cells (keratinocytes, corneocytes, fibroblasts: epidermis and dermis) and other additive factors in a dermal matrix (scaffold-structure) (hydrogel).

The respective functionality of the tissue must be checked over a wide range of external conditions (pressure, temperature, exposure to the atmosphere, etc.) and its coupling without collateral effects after performing the 
implant of the tissue. These conditions must also be verified in vitro. Another technique used to improve tissue functionality is the two photon polymerization $(2 \mathrm{PP})^{3}$ technique. The bioprinting technique consists of 3 stages: software development (with the use of computers) for the tissue or organ design and the control of cell deposition, printing (cell and hydrogel deposition), and maturation of the tissue or organ (incubation or the use of a bio-reactor). This last stage involves the use of biological techniques of cell assembly, fusion or aggregation ${ }^{3}$. Then, the respective implantation of the tissue in the skin would be performed. It is also possible to perform the implantation of the tissue in the skin avoiding the incubation process (clinical bioprinting).

Software development is the phase that includes all the planning details which proceed to the production of tissue printing. It includes images of the anatomical structure of the tissue (by means of CT (computed tomography), MRI (magnetic resonance imaging), etc.). Then, a CAD (Computer Automated Design) program is used to transfer the image to a bioprinter. Among the possible softwares that can be used are: AutoCAD, SOLIDWORKS, CATIA, etc. These programs convert the image into a blueprint. The blueprint is converted to a heterogeneous model where the materials to be used and the cellular composition and distribution are described ${ }^{5}$.

These programs transform the image into 2D cross sections (layers) of appropriate scale (adequate resolution). Then, the device can add them in a layer by layer way ${ }^{4}$. The printing stage involves a specific printing method and the use of a combination of materials or other additives ${ }^{4}$. It consists of the simultaneous deposition of cells using a deposition technique in a layer-by-layer mode ${ }^{5}$. The last stage involves the incubation process or the use of a bioreactor to grow the cells and the maturation process before the tissue is implanted ${ }^{3,5}$. However, most current bio-reactors are not appropriate to recreate the functioning of the tissue after the maturation process. Thus, it is necessary to refine the bio-reactor technology or use another incubation technique ${ }^{4}$. As it was said before, it is possible to perform the tissue implantation without incubation (performed in the operating room, clinical bio-printing).

The aggregation of cells (keratinocytes, corneocytes, fibroblasts) in hydrogel is currently under study. It is thought that the hydrogel improves the assembly of tissues and organs ${ }^{3}$.

\section{Tecnología 3D Printing}

The different types of 3D technology for printing are the following: 3D bioprinting inkjet, 3D bioprinting microextrusion, 3D bioprinting laser and within the laser technique we have the stereolithography ${ }^{4}$. These types of printers are not in competition but their application depends of the required scale. Thus, if it is for a basic research for the cell analysis and without tissue construction, laser printing is indicated ${ }^{2}$.

Inkjet 3D bioprinting: Early studies used a modified 2D inkjet printer to print layered tissues. It is a non-contact technique that uses thermal forces, piezoelectric, pneumatic or electromagnetic microvalves to print bioink drops to a substrate. Therefore, it is possible to replicate a design made on the computer ${ }^{4}$. The thermal inkjet printer is the most used. The printing cells are heated for $2 \mu$ s and thus the local temperature of $300^{\circ} \mathrm{C}$ is not a problem ${ }^{4}$ In addition, the maintaining of a thermal inkjet printer is easier than piezoelectric printer. Thus, the cells and biomaterials are modeled towards certain substrates (structures) in the form of drops ${ }^{5}$. The structure is formed by 
means of the continuous deposit of many drops in specified locations by using the software on the computer. The duration of local heating in each printing drop is for a short period of time $(2 \mu \mathrm{s})$ and the temperature increase is around 4 to $10^{\circ} \mathrm{C}$. Research has shown that it does not affect the cell viability. One of the advantages of this technique is the low cost and high spatial resolution. For other hand, one of the disadvantages is the drop size (resolution) compared to other techniques ${ }^{4}$. However, this technique also reports an impression of low viscosity and cell distortion. The viscosity can be minimized using biomaterials based on water ${ }^{2}$.

Main characteristics: Electromagnetic, thermal, or piezoelectric forces that emit successive drops of bioink towards a substrate, high speed, availability, low cost, loss of precision in the location of the drops and size, necessity for low viscosity, cell viability around $85 \% 4$.

Microextrusion 3D bioprinting: It uses mechanical or pneumatic forces to print the bioink material. The printing is by contact by means of small continuous flows of bioink material. Likewise, the printing is achieved through a CAD design (software) by using the computer. At present, it is the most used and the most common method ${ }^{4}$. The printing is of high viscosity such as complex polymers. Besides, the printing results in high cell density. However, a disadvantage is the cell distortion (due to the tensions among the cells), low resolution and loss of cell viability ${ }^{4}$.

Main characteristics: Mechanical or pneumatic forces that dispense bioink through an injector, high viscosity of bioink, high cell density, distortion of cell structure, $40 \%$ cell viability, not expensive ${ }^{4}$.

Laser 3D bioprinting: It is a non-contact technique, which is used as for example in the manufacture of chip and integrated $^{4}$. This technique sends laser pulses through a tape containing bioink. The bioink and the cells are suspended at the bottom of the tape. Then, they are vaporized by the laser pulse. It results in discrete drops which adhere to the substrate. This step is repeated several times until the 3D structure is created ${ }^{4}$. One of the main advantages of this technique is the high degree of precision and resolution (one cell per drop). It gives rise to the possibility of printing DNA and microcellular arrangements ${ }^{4}$. This technique also allows printing with a very high cell density and high resolution. Thus, for example, a bioink concentration of $10^{8}$ cells $/ \mathrm{ml} \mathrm{can}$ be used to print discrete drops containing at least one cell ${ }^{4}$. The disadvantages are the low cell viability compared to other techniques and the preparation time of the tape. However, the printing time is fast if the preparation time of the tape is not taken into account ${ }^{4}$.

Main characteristics: Bioink and the cells are suspended at the bottom of a tape and when they are vaporized by a laser pulse, they are dispensed to a substrate, high degree of precision and resolution, ability to use high viscosity, printing with high cell density, large printing time, high cost, $95 \%$ cell viability, high cost $^{4}$.

Laser 3D Stereolitografía: This technique uses photopolymerization, where the laser or ultraviolet light is directed towards a photopolymerizable liquid polymer, which converts the polymer into a layer. Since each layer is polymerizable, it is repeated several times to form the $3 \mathrm{D}$ structure ${ }^{4}$. When acrylics and epoxies are used as a photopolymerizable material, they result in high manufacturing accuracy compared with other techniques. However, the disadvantages are the intense ultraviolet radiation that is needed, the slow post-incubation-maturation process that is required and the few compatible materials for use with this technique ${ }^{4}$. 

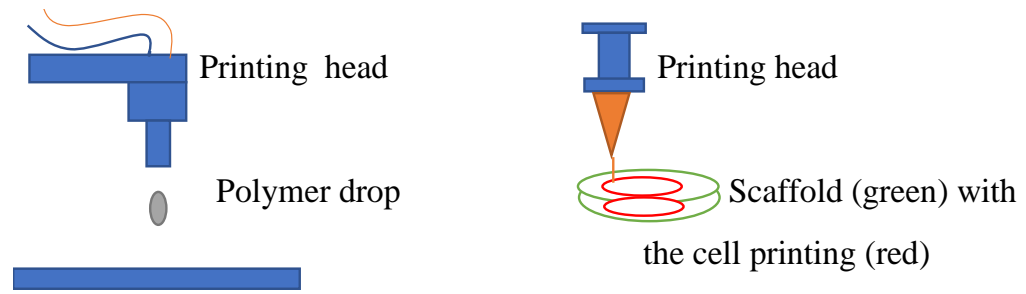

Fig.4. Inkjet printing, Extrusion printing ${ }^{5}$

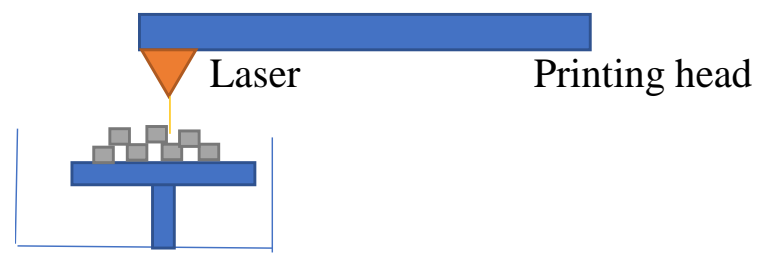

Fig.5. Laser printing

Main characteristics: It use digital light to dispense the bioink in a layer form, enough accuracy, low printing time, high intensity ultraviolet light, slow post-processing (incubation), loss of material compatibility, $90 \%$ cell viability, not expensive ${ }^{4}$.

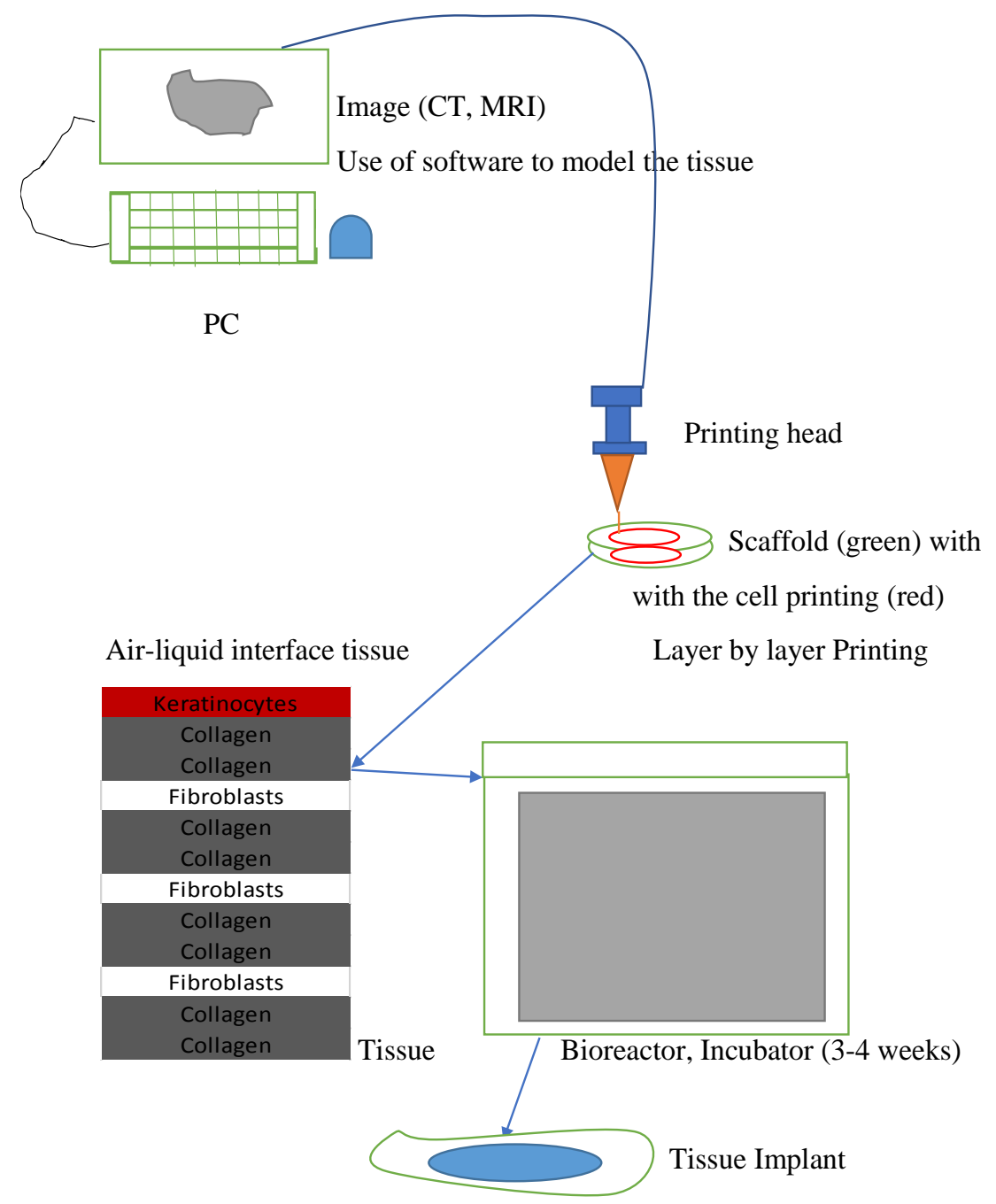

Fig.6. 3D bioprinting schematic 


\section{Printing Materials}

The characteristics that the printing materials should have are the following: appropriate viscosity, high mechanical forces, the structure should not collapse during the deposition process layer by layer, biocompatibility, biodegradable ${ }^{5}$. The biomaterials can be classified in synthetic and natural. The synthetics have the disadvantage of the cell proliferation and differentiation, while the natural biomaterials are mechanically weak ${ }^{5}$. The polyethylene glycol is one of the most used synthetic polymers for printing ${ }^{5}$. It is a water soluble polymer widely used for cell encapsulation ${ }^{5}$. The polyethylene glycol (PEG) can be modified with acrylic groups such as diacrylic ethylene glycol (PEGDA). For other hand, a copolymer based on polyte methacrylamide lactate and PEG was developed as bioprinting material ${ }^{5}$. The disadvantage of PEG is that it is a non-biodegradable polymer ${ }^{5}$.

The biodegradable synthetic polymers are for example: polylactic acid (PLA), polylactic-lactic-glycolic acid (PLGA) and poly-e-caprolactone (PCL) $)^{5}$. Among the natural biomaterials, the collagen is an important protein of the extracellular matrix $(\mathrm{ECM})^{5}$. The collagen consists of three amino acids: glycine, proline, and hydroxyproline. The cells can adhere and proliferate in the collagen. It has been widely used to regenerate skin, bone, cartilage ${ }^{5}$ and widely used for 3D printing. The disadvantage is the weak mechanical force and long-lasting coupling (cohesion) of the tissue ${ }^{5}$. The gelatin is a derivative of native collagen. However, the gelatin is not stable at the body temperature. The gelatin can be modified by adding methacrylate group to the gelatin $\operatorname{gel}^{5}$ (GelMA). GelMA hydrogels have been printed to create cartilage and cardiovascular structures ${ }^{5}$. For other hand, hyaluronic acid (HA) has a high biocompatibility and biodegradability ${ }^{5}$. HA hydrogels can be formed through the modification with the metracrilate group when they are exposed to ultraviolet light. It has a strong mechanical force ideal for printing hard tissues such as bones ${ }^{5}$.

Fibrin is a natural protein. Fibrin gels have been printed to improve the vascularization. Vascular networks, tubular tissues are printed by using fibrin gels ${ }^{5}$. Alginate is a natural polysaccharide (algae) and it is biodegradable and bioinerte ${ }^{5}$. It is excellent for printing long tissues ${ }^{5}$. For other hand, most hydrogels are weak. Thermoplastics can be deposited with these biomaterials during printing to improve the mechanical strength of hydrogels ${ }^{5}$. Examples of thermoplastics are hydroxymetiglicolide-co-e-caprolactone added with a methacrylate group (through photo-polymerization) ${ }^{5}$. Therefore, the resistance to axial and rotational forces was improved ${ }^{5}$. The cells removed from tissues and organs (by using physical or biological methods) (de-cellularized matrix, dECM), is an excellent material for printing since they have bioactive signals from native tissues. These cells mimic the composition of tissues 5 .

Ceramic or glass particles can be incorporated to be added to the scaffold of the matrix. These particles mixed with hydrogels reinforce the mechanical forces. Thus, it is improved the bioactivity ${ }^{5}$.

\subsection{Bioink}

This material is printed layer by layer during the bioprinting process. It is made of cellular materials, additives (growth factors, molecules, etc.) and a support scaffold (extracellular matrix) ${ }^{4}$. Among the different bioink components used for bioprinting are: synthetic scaffolds, natural scaffolds, hydrogel scaffolds ${ }^{4}$. 
The specific properties of a bioink depend of the mode of printing, the type of tissue and the cell concentration for a given bioink. For example, inkjet prints require low viscosity, low thermal conductivity and low temperature. For other hand, extrusion prints can withstand high viscosities but it presents the problem of possible cell distortion ${ }^{4}$. A balance must be taken between obtaining the respective structural properties required without affecting the cell viability ${ }^{4}$.

Therefore, there is an optimal concentration range of bioink, where the increase of the cell concentration can affect the cell viability affecting the cell migration and diffusion ${ }^{4}$.

\section{Bioink scaffolds}

It should provide cells with secure fixation and protection from the mechanical and thermal stresses of the printing. It should also support cell growth and proliferation without affecting the cell phenotype ${ }^{4}$.

The biocompatibility is the biggest limiting factor for the scaffolds. They should be cytocompatible without causing immune response, inflammatory response or premature differentiation of the cell stem ${ }^{4}$.

\section{Hidrogels scaffolds}

It is currently one of the most used. They can mimic the extracellular environment of the tissue and can absorb water ${ }^{4}$. These scaffolds are moldable polymers and they have been produced from a wide range of components such as collagen, fibrin, algae and some other materials ${ }^{4}$.

For example, it has been applied in contact lenses and biological adhesives such as polyethylene glycol polymer ${ }^{4}$. It is also used to form aortic valve ducts. There are two techniques regarding their use: they can be printed firstly, and after the cells are deposited (keratinocytes, corneocytes, fibroblasts), or they can be printed with the cells already suspended in the hydrogel matrix ${ }^{4}$. For example, a bionic ear could be constructed by means of the hydrogel matrix by placing algae (alginate) with corneocytes around an inductive coil antenna. Therefore, it could receive electromagnetic signals over a wide frequency range ${ }^{4}$.

Main characteristics: It is composed of hydrophilic polymers linked through covalent bonds or through intermolecular attractions. The hydrophilicity allows easy exchange of gases and nutrients, high biocompatibility, and easily modifiable. Besides, it has low mechanical properties and cell culture (sediment). The viscosity is adjustable by adding other factors ${ }^{4}$.

Synthetic scaffolds: Examples of synthetic scaffolds include polyethylene glycol such as PEG diacrylate and polyacrylamide gels ${ }^{4}$. The advantage of these bioinks is the ability to manipulate their physical and chemical properties $^{4}$. One of the disadvantages is the low cellular interaction. They synthetic scaffolds also do not effectively mimic the biological environment of the tissue ${ }^{4}$.

Main characteristics: It is derived from natural and synthetic sources such as polyethylene glycol (PEG), PEG diacrilate (PEGDA) and polyacrylamide gels (PAAm). The synthetic scaffold is easily modifiable, composed of functional groups and presents non-immunogecity. It does not have a good cell coupling. Besides, PEG has low viscosity and pluronic acid has high viscosity ${ }^{4}$. 
Natural scaffolds: These scaffolds include polymers such as gelatin, collagen, fibrin, algae (alginate) and other natural polymers ${ }^{4}$. They have the advantage of their high cell viability, biocompatibility and cell growth compared to synthetic bioinks. A disadvantage is that natural bioink does not support remodeling and elasticity compared to synthetic bioink ${ }^{4}$. For example, gelatin and aginate have low resolution ${ }^{4}$. However, natural and synthetic bioink compounds can be formed to improve the resolution ${ }^{4}$.

Main characteristics: It is made with biological materials such as collagen, fibrin, hyaluronic acid. It is also highly biocompatible with limited Modification. Gelatin and fibrinogen have low viscosity while hyaluronic acid has high viscosity and silk has high viscosity ${ }^{4}$.

A resume of the materials used for the biofabrication of tissues and organs through $3 \mathrm{D}$ bioprinting ${ }^{5}$ is as follows:

Tissue construction.- Biomaterials: Alginate, gelatin, agarose, calcium salts of polyphosphate; Cells: sarcoma cells of human osteogenic; Applications: high rate of cell proliferation, Young's modulus reduced.

Scaffold of osteochondral tissue.- Biomaterials: PCL, alginate; Cells: osteoblasts, chondrocytes; Applications: mechanical properties improved, high cell viability.

Osteochondral models.- Biomaterials: Gelatin, methacrylamide and PLA microcarries; Cells: chondrocytes, osteoblasts; Applications: better mechanical properties, high cell viability.

Atrial cartilage.- Biomaterials: Collagen; Cells: chondrocytes; Applications: specific morphology of the patient, live neocartilage formation.

Human skin.- Biomaterials: Collagen; Cells: fibroblasts and keratinocytes; Applications: Good retention, high cell viability, good morphological and biological imitation of the human tissue.

Aortic valve.- Biomaterials: PEGDA + alginate; Cells: aortic pig VICs; Applications: high cell viability, heterogeneous mechanical forces. Biomaterials: alginate-gelatin; Cells: aortic pig VICs, human aortic roots SMCs; Applications: high cell viability, phenotype retention, good cell dispersion. Biomaterials: Metacrilate HA and GelMA; Cells: HAVICs; Applications: high cell viability.

Bionic ear.- Biomaterials: alginate + chondrocytes (ear), AgNP-silicone (antenna-coil); Cells: Chondrocytes; Applications: high cell viability, neocartilage formation.

Similar tissues.- Biomaterials: Decellularized cartilage tissues, heart tissues, adipose tissue; Cells: hASCs, hTMSCs; Applications: high cell viability, ECM formation.

Neural cellular structures.- Biomaterials: collagen, fibrin gel; Cells: Hippocampal and cortical cells, NTw neuronal cells; Applications: Healthy cellular electrophysiological structures.

\subsection{Differentiation of the stem cell in the printing}

One of the biggest advantages of 3D printing is the ability to influence the differentiation of the cell stem in the multiple stages of the process ${ }^{4}$. Thus, the selection of the source of the cell stem, printing method, scaffold, additive factors, incubation process can influence the differentiation of the cell stem towards a specific target of the 
tissue $^{4}$. The use of a cell stem also influences the immunotolerance and expansion once it is implanted in the tissue ${ }^{4}$.

Sources of cell stem.- The three main sources used are the following: embryonic cell stem (high degree of multipotence but it has ethical and immunogenicity aspects), mesenchymal cell stem (it stimulates the immunotolerance with low degree of multipotence), and pluripotent cell stem (it has a high degree of multipotence but it promotes tumorigeneis according to some studies $)^{4}$.

Printing method.- The tensions in each printing technique can influence the differentiation of the cell stem ${ }^{4}$. For example, the mechanical pressure in the inkjet printing influences the differentiation of the mesenchymal cell stem to form cartilage and bones ${ }^{4}$. For other hand, shear forces in the extrusion printing influence the differentiation in endothelial tissues and bones. Laser printing preserves multipotence and it uses another method to stimulate the differentiation as mentioned previously ${ }^{4}$.

Additive factors.- These factors must be added to the bioink before the printing or to the printed tissue before the maturation process ${ }^{4}$. These factors influence the differentiation of the cell stem. Examples of these factors are: FGF, PDGF, morphogenetic proteins, etc ${ }^{4}$. Other factors that influence the differentiation of the cell stem include dexamethasone, ascorbic acid ${ }^{4}$.

Small spheres of polymers (microcarriers) have been shown to promote the differentiation when they are added to the bioink giving it adhesion and fixation ${ }^{4}$.

Biopolymers.- Hidrogels and biopolymers are not always suitable for printing methods. One of the options is to combine substances to maximize the usefulness of polymers ${ }^{4}$. For example, Atala and his colleagues included polymers to a scaffold hydrogel containing progenitor cells. Thus, it was possible to incorporate tri-calcium phosphate into gelatin and hyaluronic acid to the bioink for bone printing ${ }^{4}$.

It is necessary to improve the efficiency of the printing process and the selection of biopolymers. Therefore, it is possible to enhance the cell density without cell distortion after printing.

Vascularity.- One of the biggest complications is the creation of vascular networks in the implantation of tissues to achieve its functionality. It is very important for the delivery of nutrients and the expulsion of sweat or waste. Thus, it contributes to the formation of complete tissues. The size of the vascular networks is around $100-200 \mu \mathrm{m}^{4}$. For other hand, the printing of capillary vessels (vascularity) has limitations in terms of resolution and speed ${ }^{4}$. The capillary vessels must have a dimension of $3 \mu \mathrm{m}$ while the highest resolution of a laser printer is $20 \mu \mathrm{m}^{4}$. In addition, the printing time is important for the cell viability.

Scaffold fibrin (dermis) and human microvascular endothelial cells have been used for vascularity ${ }^{2}$.

One of the options is to perform vascularization live by incorporating angiogenic growth factors and bioinks. Therefore, it improves the vascularization growth ${ }^{4}$. Vascular network of synthetic origin ${ }^{4}$ can be used as another alternative. It is under investigation the development of vascular network which allow the functionality of the implanted tissue. 
One of the techniques is to create sunken channels in the hydrogels through $3 \mathrm{D}$ printing ${ }^{5}$.

\section{Clinical 3D Bioprinting}

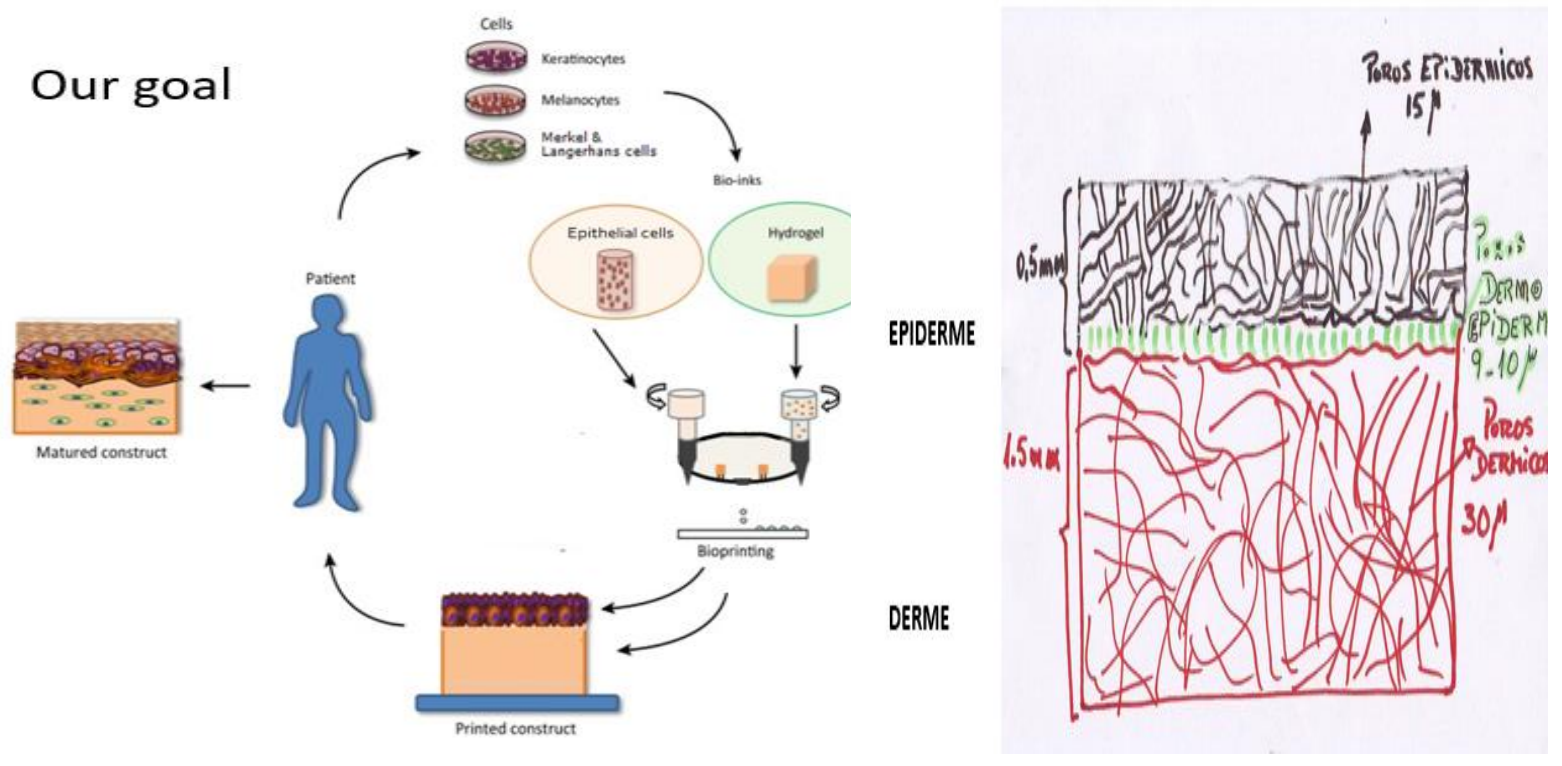

Fig.7. Epidermis and Dermis ${ }^{30}$

Live cell culture is much faster. It is a pore technology obtained with a 3D bioprinter. It allows regulating the porosity of the dermis and epidermis. Therefore, it is possible to orient the cell according to its size and shape ${ }^{30}$.

Dermis-Epidermis cells are obtained with a biopsy of the Patient $(3 * 2 \mathrm{~cm})$. Then, an enzyme is placed to perform dermoepidermal separation. Thus, the epidermal cells are separated and isolated in a medium enriched with platelets from the patient ${ }^{30}$.

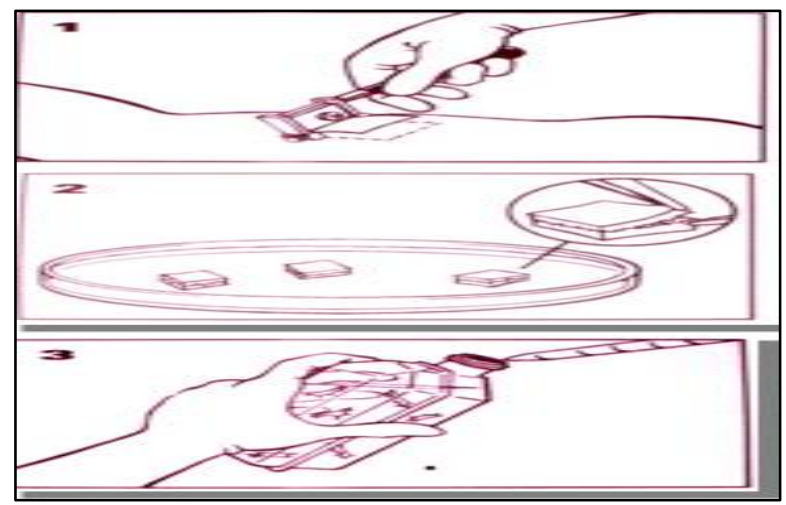

Fig.8. Biopsy of the patient ${ }^{30}$

Then, the gel preparation is carried out with collagen, hyaluronic acid and chitosan. Subsequently, the patient's cells and their platelet-enriched plasma are placed. The dermis and epidermis are printed with the gel and the cells according to the desired size and thickness, and to the case of the patient ${ }^{30}$.

Then, the gel preparation is carried out with collagen, hyaluronic acid and chitosan. Subsequently, the patient's cells and their platelet-enriched plasma are placed. The dermis and epidermis are printed with the gel and the cells according to the desired size and thickness, and to the case of the patient ${ }^{30}$. 


\section{MJBAS}



Fig.9. Gel preparation ${ }^{30}$

The printer is a sterile flow printer with adjustable temperature that allows rapid drying of the skin sheets. The dermis is done of collagen and chitosan and hyaluronic acid. The epidermis is constituted of the same plus epithelial cells and platelet rich plasma of the patient. The ink can be improved to maintain the viability and proliferation of the epidermal cells during printing ${ }^{30}$.
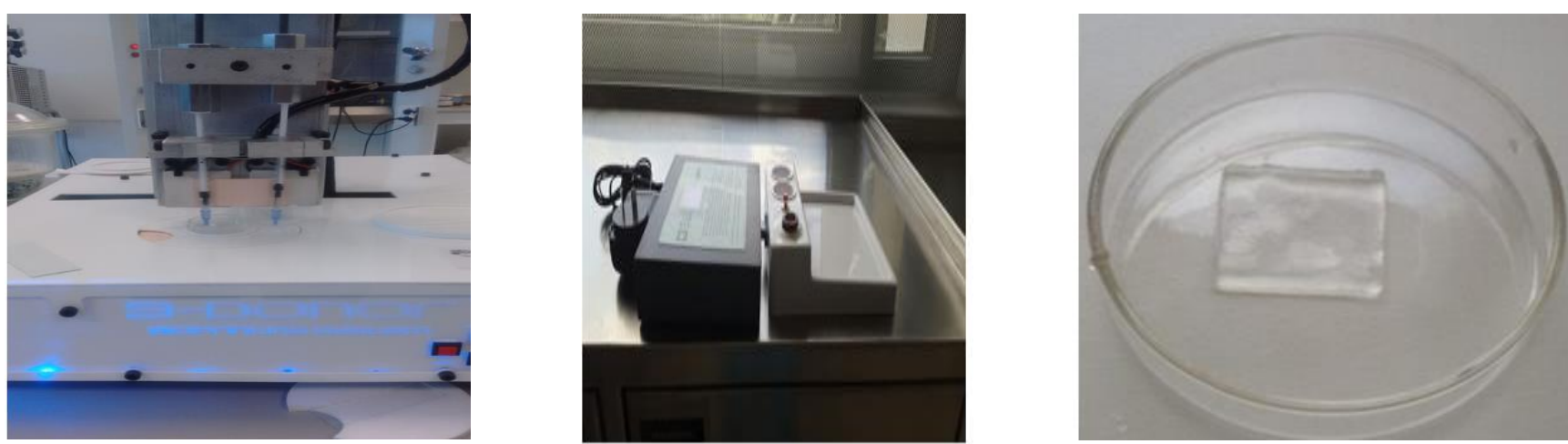

Fig.10. 3D Bioprinter and the gel obtained

For other hand, there are also the hyalomatrix for superficial implants. It is a silastic (superficial) membrane with hyaluronic acid fibers (deeper). Besides, there is also the prompt sheet: $10 * 10 \mathrm{~cm}$ esterified hyaluronic acid membrane. It uses the technology of micropores of 40 microns. The pore technology is essential for vascularization and waste disposal and interconnectivity. The keratinocytes, fibroblasts and endothelial cells need pores of different sizes ${ }^{30}$.

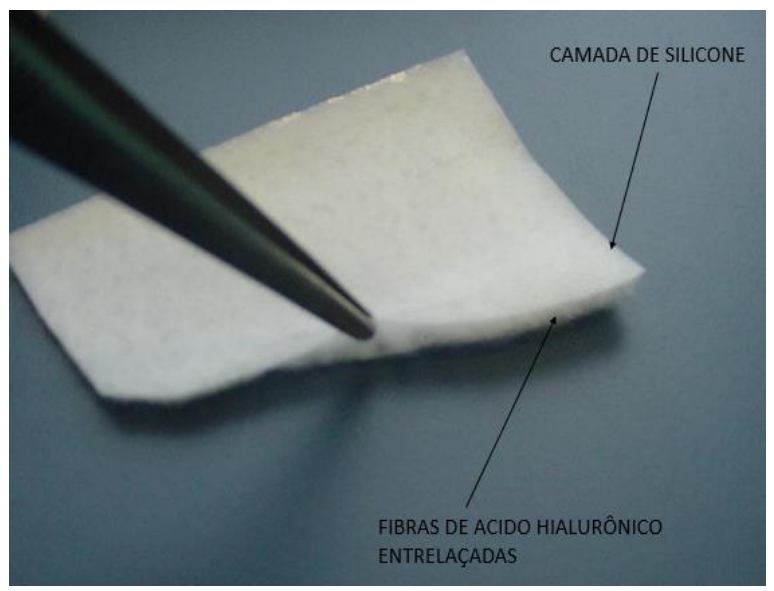




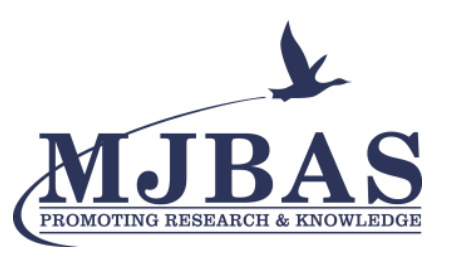

Mediterranean Journal of Basic and Applied Sciences (MJBAS)

Volume 5, Issue 1, Pages 40-56, January-March 2021



Fig.11. Hyalomatrix ${ }^{30}$

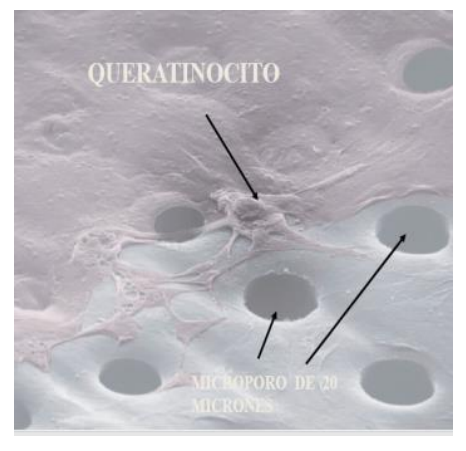

\section{Conclusions and recommendations}

The 3D printing has a lot of applications in engineering, industry and medicine. In Medicine, the most important application is the tissue and organ engineering. With respect to the tissue engineering, it has advanced much for the manufacture of skin and grafting. Also, it has important contributions for conducting scientific experimentation in the evaluation and discovery of drugs, skin disorders and diseases ${ }^{1}$.

Before the technology of 3D bioprinting, the conventional methods of tissue engineering (without 3D bio-printers) were used. Nevertheless, it had little spatial relationship between the individual elements (cells) of the desired tissue $^{4}$. With the 3D bio-printing technique, it was possible to improve both spatial resolution and reproducibility and to obtain the optimal conditions for cell incubation and maturation.

The 3D bioprinting allows the respective aggregation layer by layer of the cells and the organization of multiple types in a desired structure ${ }^{5}$. The respective cell culture is performed in vitro ( 3 to 4 weeks), allowing the respective growth and maturation to achieve the desired tissue. After, the tissue implantation is performed. The bioprinting technique consists of 3 stages: software development (use of PC, image acquisition and software) for the tissue or organ design and the control of cell deposition, printing (cell and hydrogel deposition), and maturation 
of the tissue or organ (incubation or the use of a bio-reactor). This last stage involves the use of biological techniques of cell assembly, fusion or aggregation (use of a reactor) ${ }^{3}$. Then, the respective implantation of the tissue in the skin would be performed. It is also possible to perform the implantation of the tissue in the skin avoiding the incubation process (clinical bioprinting). Thus, it is possible to avoid the incubation stage and the maturation time for the tissue ( 3 to 4 weeks). Therefore, the skin is implanted directly from the printing to the patient. Among the different bioink components used for bioprinting are: synthetic scaffolds, natural scaffolds, hydrogel scaffolds ${ }^{4}$. Respect to the printing techniques, there are the following: 3D bioprinting inkjet, 3D bioprinting microextrusion, 3D bioprinting laser. The microextrusion 3D bioprinting is the most used for the advantages that it offers to the cell deposition and spatial resolution. In resume, 3D bioprinting is of great importance in the field of biomedicine. For example, organ transplantation is one of the biggest treatments in medicine for many organ disorders. Thus, the supply of donors is limited and the biofabrication of organs and tissues can help for the respective transplant ${ }^{5}$.

\section{Bibliography}

1.- Lee, Singh, Trasatti, Bjornsson, Xu, Nga Tran, Yoo, Dai, Karande, Designd and Fabrication of Human Skin by Three-Dimensional Bioprinting vol. 20, \#6, 2014.

2.- Gao, Cui, Three-dimensional bioprinting in tissue engineering and regenerative medicine, Biotechnol Lett, October 2015.

3.- Mironov, Reis, Derby, Bioprinting: A Beginning, Tissue Engineering vol.12, \#4, 2006.

4.- Bishop, Mostafa, Pakvasa, Luu, Lee, Moriatis, Ameer, Tong-Chuan He, Russel Reid, 3-D bioprinting technologies in tissue engineering and regenerative medicine: Current and future trends, Genes Dis. Dec 2017.

5.- Xia, Ye, Tissue and Organ 3D Bioprinting, SLAS Technology, vol. 23 (4), 2018.

6.- Yong Leng Chuan, Shivani Amish Kumar Pandya, Fabrication of Non-Implant 3D Printed Skin, MATEC Web of Conferences 152, 02016 (2018).

7.- Alfredo Gragnani, Jeffrey Morgan, Lydia Ferreira, Experimental model of cultured keratinocytes, Cir Bras 2003, Vol 18.

8.- Billiet, Vandenhaute, Schelfhout, Vlierberghe, Dubruel, A review of trends and limitations in hydrogel-rapid prototyping for tissue engineering, Elsevier, Biomaterials 33, 6020-6041, 2012.

9.- Gu Qi, Jie, YangJie, Liu, Gordon, Zhou Qi, Three-dimensional bio-printing, Science China, Life Sciences, vol.58, No. 5: 411-419, May 2015.

10.- Boland, Xu, Damon, Cui, Application of inkjet printing to tissue engineering, Biotec.., 910-917, July 2006.

11.- Brian Derby, Printing and Prototyping of Tissues and Scaffolds, Science, vol. 338, November 2012.

12.- Fu-Yu Hsieh, Shan-hui Hsu, 3D bioprinting: A new insight into the therapeutic strategy of neural tissue regeneration, Organogenesis, 11:153, 2015. 
Mediterranean Journal of Basic and Applied Sciences (MJBAS)

Volume 5, Issue 1, Pages 40-56, January-March 2021

13.- Marie-Noëlle Giraud, Anne, Géraldine Guex, Hendrik T. Tevaearal, Cell Therapies for Heart Function Recovery: Focus on Myocardial Tissue Engineering and Nanotechnologies, Cardiology Research and Practice, 2012, Article ID 971614, 10 pages.

14.- Payam Akhyari, Hiroyuki Kamiya, Axel Haverich, Matthias Karck, Artur Lichtenberg, Myocardial tissue engineering: the extracelular matrix, Elsevier, European Journal of Cardio-thoracic Surgery 34 (2008) 229-241.

15.- Steinhoff, Stock, Najibulla Karim, Mertsching, Timke, Mellis, Pethig, Haverich, Bader, Tissue Engineering of Pulmonary Heart Valve on Allogenic Acellular Matrix Conduits: In Vivo Restoraton of Valve Tissue, American Heart Association, 2000, 102, 50-55.

16.- Ott, Goh, Black, Kren, Perfusion-decellularized matrix: Using nature's platform to engineer a bio artificial heart, Nature Medicine, March 2008.

17.- Grabarcyk, Przybyszewska, Sodium Alginate/Gelatine Hydrogels for Direct Bioprinting-The Effect of Composition Selection and Applied Solvents on the Bioink Properties, Materials, August 2019.

18.- Leberfinger, Dinda, Yang Wu, Koduru, Veli Ozbolat, Ravnic, Ibrahim Ozbolat, Bióprinting functional tissues, Elsevier, Acta Biomaterialia, Januar 2019.

19.- Enrique Ortega, Andrés García, Alpha Espinoza, Carmen Escobedo, Accurate Calibration in Multi-Material 3D Bioprinting for Tissue Engineering, Materials, August 2018.

20.- Datta, Barui, Yang Wu, Veli Ozbolat, Moncal, Ibrahim Ozbolat, Essential steps in bioprinting: From pre-to post-bioprinting, Elsevier, Biotechnology Advances, 2018.

21.-Leberfinger, Ravnic, Dhawan, Ibrahim Ozbolat, Concise Review: Bioprinting of Stem Cells for Transplantable Tissue Fabrication, Manufacturing for Regenerative Medicine, Stem Cells Translational Medicine, Wiley Periodicals 2017.

22.- Ali, Hosseini, Vainio, Taïed, Cario-André, Rezvani, Skin equivalents: skin from reconstruction as models to study skin development and diseases, British Journal of Dermatology, April 2015.

23.- Fatemeh Abedini, Factors involved in Tissue Regeneration, Journal of Regenerative Medicine, July 2016.

24.- Matthew Randall, Astrid Jüngel, Markus Rimann, Karin Kozak, Advances in the Biofabrication of 3D Skin in vitro: Healthy and Pathological Models, Frontiers in Bioengineering and Biotechnology, October 2018.

25.- Gangatirkar, Paquet-Fifield, Li, Rossi, Kaur, Establishment of 3D organotypic cultures using human neonatal epidermal cells, Nature Protocols, Vol.2, No.1, 2007.

26.- Hacioglu, Yilmazer, Ustundag, 3D Printing for Tissue Engineering Applications, Journal of Polytechnic, 2018, 21 (1), 221-227.

27.- Kacarevic, Rider, Alkildani, Retnasingh, Smeets, Jung, Ivanisevic, Barbeck, An Introduction to 3D Bioprinting: Possibilities, Challenges and Future Aspects, Materials, November 2018. 
28.- Pourchet, Thepot, Albouy, Courtial, Boher, Blum, Marquette, Human Skin 3D Bioprinting Using Scaffold-Free Approach, Advanced HealthCare Materials, 2017.

29.- Rider, Kacarevic, Alkildani, Retnasingh, Barbeck, Bioprinting of tissue engineering scaffolds, Journal of Tissue Engineering, Volume 9, 1-16, 2018.

30.- Alberto Bolgiani, C.E.P.A.Q. Centro de Excelencia para la Asistencia de Quemaduras, Fundación Benaim, Hospital Alemán, Buenos Aires, Argentina. 